\title{
Auditory pathology in cri-du-chat (5p-) syndrome: phenotypic evidence for auditory neuropathy
}

\author{
D Swanepoel \\ Department of Communication Pathology, University of Pretoria, Gauteng, Pretoria, \\ South Africa \\ Tel.: +27 12 4202304; \\ Fax: +27 12 4203517; \\ e-mail: dewet.swanepoel@up.ac.za
}

\section{Figures at the bottom of the document}

\begin{abstract}
5p-(cri-du-chat syndrome) is a well-defined clinical entity presenting with phenotypic and cytogenetic variability. Despite recognition that abnormalities in audition are common, limited reports on auditory functioning in affected individuals are available. The current study presents a case illustrating the auditory functioning in a 22-month-old patient diagnosed with 5p- syndrome, karyotype 46,XX,del(5)(p13). Auditory neuropathy was diagnosed based on abnormal auditory evoked potentials with neural components suggesting severe to profound hearing loss in the presence of cochlear microphonic responses and behavioral reactions to sound at mild to moderate hearing levels. The current case and a review of available reports indicate that auditory neuropathy or neural dys-synchrony may be another phenotype of the condition possibly related to abnormal expression of the protein $\beta$-catenin mapped to $5 \mathrm{p}$. Implications are for routine and diagnostic specific assessments of auditory functioning and for employment of nonverbal communication methods in early intervention.

$5 \mathrm{p}$-(cri-du-chat syndrome) is one of the most common chromosome deletion syndromes with a reported incidence between 1:15,000 and 1:50,000 live births $(1,2)$. The prevalence in mentally retarded persons is reported to be as high as 1:350 (1). Although it is a well-defined clinical entity, with some phenotypic and cytogenetic variability, there is a dearth of published reports on the auditory functioning of individuals presenting with a partial deletion of the short arm of chromosome 5 . The majority of reports on genotype-phenotype correlations in 5p- syndrome and of developmental and behavioral profiles do not consider hearing loss as a clinical feature $(1,3-8)$. Reports by Cornish and Pigram (7) do, however, consider an auditory behavioral phenotype, hyperacusis, as a characteristic trait. Hyperacusis is a condition characterized by a hypersensitivity to sound, which causes auditory discomfort, and is reported to be one of the main characteristics of the syndrome (7).
\end{abstract}

Published reports concerning hearing loss are scarce. Cerrutti Mainardi (4) in reviewing management options briefly comments that sensorineural hearing loss is characteristic of some patients. Another report cites that $20 \%$ of individuals with $5 \mathrm{p}$ - syndrome present 
with hearing loss but this figure is unsubstantiated (9). A multiple case report by Baccichetti et al. (10) report the presence of a mild bilateral high-frequency sensorineural hearing loss in several affected members of one family. Another separate case study in the report by Baccichetti et al. (10) indicates a similar bilateral high-frequency sensorineural hearing loss but of a severe degree. These authors suggest the location of a possible gene involved in cochlear development located in 5p (10). A more recent case study by Choong et al. (11) also reported a newborn hearing screening failure on both otoacoustic emission (OAE) and automated auditory brainstem response (ABR) tests for a case of cri-du-chat syndrome. Although these screening results strongly infer the presence of a hearing loss, the results from a diagnostic evaluation was not reported.

The current study presents a case illustrating the auditory functioning in a patient with $5 \mathrm{p}$ - syndrome according to electrophysiological and behavioral assessment. The results are compared with available reports to delineate and contextualize the phenotypical auditory characteristics more precisely within the existing body of literature. The results have important implications for the nature of auditory functioning in such cases and for optimal treatment options.

\section{Materials and methods}

A 22-month-old female subject diagnosed with 5p-syndrome (cri-du-chat) was referred for a behavioral and electrophysiological evaluation of auditory functioning at the Hearing Clinic, Department of Communication Pathology, University of Pretoria. Diagnosis of 5p- syndrome was made after birth based on typical clinical features such as facial dysmorphisms, transverse flexion creases, and hypotonia in combination with the characteristic cat-like cry at birth (4). This clinical diagnosis was subsequently confirmed by karyotype analysis at 2 months of age and indicated a terminal deletion in $5 p$ presenting with a 46,XX,del(5)(p13) karyotype.

The patient was born at 40 weeks gestational age after an uneventful pregnancy weighing $2220 \mathrm{~g}(<10$ th percentile). No hospitalizations or operations were necessary after birth. Her motor milestones were delayed with the ability to sit without support emerging at 16 months and being able to stand with support at 20 months. She could not yet walk at 22 months of age when assessments were conducted. Her communication abilities were very limited, and she did not utilize words or vocalizations to convey communication intention.

An electrophysiological test battery was conducted on two separate visits, and a third visit was required for a behavioral assessment. The electrophysiological assessment of audition included tympanometric measurements of middle-ear functioning; diagnostic distortion product otoacoustic emissions (DPOAE) for measuring outer hair cell integrity of the cochlea (750-8000 Hz); click-evoked ABR measurements to assess the neural response to sound of the cochlea, eighth nerve and lower brainstem and; auditory steady- 
state responses (ASSR) to assess frequency-specific neural synchrony for estimating hearing thresholds $(0.5,1,2$, and $4 \mathrm{kHz})$. Insert earphones were used for presentation of all stimuli and click stimuli were presented in both condensation and rarefaction polarities for ABR recordings. She was lightly sedated for electrophysiological assessments with chloral hydrate $(5 \mathrm{ml} / 10 \mathrm{~kg})$.

The behavioral assessment of hearing was conducted using a visual reinforcement paradigm in a free-field environment within a soundproof booth. Behavioral observation was also employed for responses to tonal stimuli and to cold running speech.

\section{Results}

Type A tympanograms indicating normal middle-ear functioning, and the absence of any middle-ear effusion were measured in both ears. Once asleep diagnostic DPOAE measurements, which are acoustical measurements of the mechanical integrity of the outer hair cells, were recorded from both ears and revealed no emissions at any frequencies.

Subsequently a click-evoked ABR assessment was conducted in both ears. The results are presented in Figs 1 and 2. Recordings from both ears indicated grossly abnormal neural responses at high intensities, which disappeared at $85 \mathrm{~dB}$. A neural component, which could not be identified as either wave III or wave V, was present at high intensities at a latency between 4.6 and $4.8 \mathrm{~ms}$ for both ears. A prominent finding in both ears, however, was the presence of a cochlear microphonic (CM) response. The response is cochlear in origin and was identified by reversing stimulus polarity from rarefaction to condensation. This revealed inverting positive- and negative peaks initiating after the stimulus until approximately $3 \mathrm{~ms}$ at high intensities. This ringing CM response was recorded in both ears at decreasing intensities up to $85 \mathrm{~dB}$ in the right ear and $90 \mathrm{~dB}$ in the left ear. To control the biologic origin of the response, as opposed to an electrical artifact trial, was recorded at the same intensity but with the insert earphone tube clamped for the duration of the recording. No artifactual response was visible, and therefore the recorded response was biologic in nature representing a $\mathrm{CM}$.

An ASSR assessment was subsequently conducted to assess frequency-specific thresholds. Initial presentation of dichotic multiple modulated tones $(0.5,1,2$, and $4 \mathrm{kHz})$ commenced at $60 \mathrm{~dB}$ hearing level. The patient indicated an immediate behavioral response to the stimuli at this intensity and awoke from her sedated sleep. A second assessment was scheduled for the following week to complete the evaluation. The second assessment utilized single modulated tones presented monaurally to avoid a similar behavioral reaction. The right ear presented no responses across frequencies at a maximum stimulation intensity of $90 \mathrm{~dB}$, while the left ear only indicated two significant response thresholds: $80 \mathrm{~dB}$ at $1000 \mathrm{~Hz}$ and $90 \mathrm{~dB}$ at $2000 \mathrm{~Hz}$. 
The subsequent behavioral assessment could not reveal hearing threshold values with visual reinforcement audiometry as she could not condition to the task. Minimum response levels were, however, observed between 40 and $60 \mathrm{~dB}$ across frequencies $(0.5$, $1,2$ and $4 \mathrm{kHz})$ with the poorer responses $(60 \mathrm{~dB})$ observed at the higher frequencies of 2 and $4 \mathrm{kHz}$. A speech awareness minimum response level was also observed at $40 \mathrm{~dB}$, which confirms the range of minimum response levels with tonal stimuli in the free field.

\section{Discussion}

The abnormal ABR recordings, with neural components only at maximum intensities indicative of severe to profound hearing loss, in the presence of CM responses and in addition to the behavioral reaction to sound at mild to moderate hearing levels is clearly characteristic of auditory neuropathy. This term was first coined in the literature as the clinical entity representing these diagnostic findings in 1996 (12). Diagnosis is made on the basis of absent or atypical ABR recordings in the presence of OAEs and/or a CM response (12). These individuals present with hearing losses of variable severity and configuration with speech understanding 'worse' than would be predicted from the audiogram. The significant variability in degree to which diagnostic markers occur indicates the heterogeneous nature of auditory neuropathy with several sites of possible pathology (13).

The abnormal neural responses measured by the ABR in the current case suggest a dyssynchronous discharge of neural activity along the vestibulocochlear nerve while the presence of a CM indicates residual cochlear integrity. As electrophysiological techniques, such as the ABR and ASSR, rely on neural synchrony to generate responses, these measures cannot predict actual hearing thresholds. A study on ASSRs in cases of auditory neuropathy has previously indicated absent or elevated responses, which correspond with the results obtained in the current case (14). The mismatch of electrophysiological and behavioral responses to sound is therefore clear in the current case. Behavioral and even the electrophysiological assessment when the patient awoke during a sedated sleep from stimuli presented at $60 \mathrm{~dB}$ indicates better thresholds for sound awareness than that predicted by the minimum electrophysiological response levels.

This report is the first to associate auditory neuropathy with the 5p-syndrome. Reports on auditory functioning for the syndrome are, however, scarce and have mostly been from parental account and none have reported diagnostic electrophysiological assessments for assessing auditory functioning $(3,7,15)$. The only case of electrophysiological assessment of auditory functioning is a screening OAE, and automated ABR conducted on a 6 weeks old infant with 5p-syndrome. Results indicated similar findings as diagnostic results in the current case with a failed screen on both procedures (11). 
The case studies by Baccichetti et al. (10), which report hearing loss in several cases of $5 \mathrm{p}$ - syndrome, determined hearing status by pure-tone audiometry. Results indicated bilateral mild hearing loss sloping toward the high frequencies. As electrophysiological procedures were not conducted, auditory neuropathy cannot be dismissed as it may occur with varying degrees of sensitivity loss including normal sensitivity. Interestingly, the authors report that the individual under investigation presented with major difficulties in speech comprehension compared with his performance ability (10). This is typical of auditory neuropathy with speech understanding 'worse' than would be predicted from the audiogram (13). The dys-synchrony of neural activity associated with auditory neuropathy results in distortion of the auditory signal, which makes speech discrimination very difficult.

The complaint of poor verbal communication abilities especially in expressive language is, however, cited as a common phenomenon in 5p- syndrome even in reports where hearing loss was not mentioned. Initially, the cause for this was attributed predominantly to cognitive delay but as more recent reports have emerged indicating significantly better cognitive outcomes an increasing uncertainty has been raised to what exact causative factors are attributing to the poor verbal communication abilities $(6,8,15,16)$. It is probable, therefore, that auditory neuropathy or neural dys-synchrony, as observed in this case and possibly even in the case presented by Baccichetti et al. (10), may be attributing to some of the characteristically poor verbal communication abilities. The fact that this is observed even in cases where hearing loss has not been reported may indicate a continuum of severity in neural dys-synchrony depending on the site and size of the genetic deletion or a lack of diagnostic specificity for auditory neuropathy or even both. A deletion in 5p13, as diagnosed in the current case, has previously been reported to present with a particularly severe degree of clinical features (4).

These findings are further supported by a growing body of recent studies indicating the benefit which individuals with 5p- syndrome experience with early use of non-verbal communication methods, such as sign language or alternative augmentative techniques, to facilitate expressive communication $(6,15,16)$. This is similar to reports of children with auditory neuropathy for whom the introduction of a non-verbal communication method in combination with speech is recommended to facilitate communication because of the poor discrimination of speech $(17,18)$.

A possible reason for the genotype-phenotype correlation of these auditory findings may be related to the abnormal expression of a protein involved in cell adhesion and transduction of intercellular signals, $\beta$-catenin. Medina et al. (19) recently reported that $\beta$ catenin is genetically mapped to the $5 \mathrm{p} 15.2$ region and genetic deletion thereof correlates to the mental retardation phenotype of 5p-syndrome. Recent studies have also, however, linked $\beta$-catenin to cell proliferation in developing auditory epithelia and more specifically in the structural maturation of the organ of Corti as investigated in mice and rats (20-22). Abnormal expression of $\beta$-catenin may therefore not only be responsible for the phenotype of mental retardation but also for the phenotype of auditory functioning. This is in agreement with the proposal by Baccichetti et al. in 1988 (10) that a gene involved in cochlear development may be mapped to chromosome 5p. Further 
investigation into the variability of auditory functioning in these individuals using electrophysiological techniques with high diagnostic specificity is necessary along with consideration of the possible effect of $\beta$-catenin expression in $5 p$ - syndrome.

Results of the current case study, in light of available reports, suggest that auditory neuropathy or neural dys-synchrony may represent another $5 p$ deletion phenotype. Clinical diagnosis will require routine audiological assessments utilizing an electrophysiology and behavioral test battery. A management implication in such cases is the initiation of non-verbal communication methods for early intervention to allow optimal language acquisition and communication competence and conservative amplification. 
Fig. 1. Right ear ABR recordings: the top recording is the trial run to ensure that responses were not because of stimulus artifacts. The recordings below these indicate the presence of the cochlear microphonic response, which inverts with changing polarity (rarefaction and condensation). A neural response is observed at $95 \mathrm{~dB}$ but cannot be identified as either wave III or V.

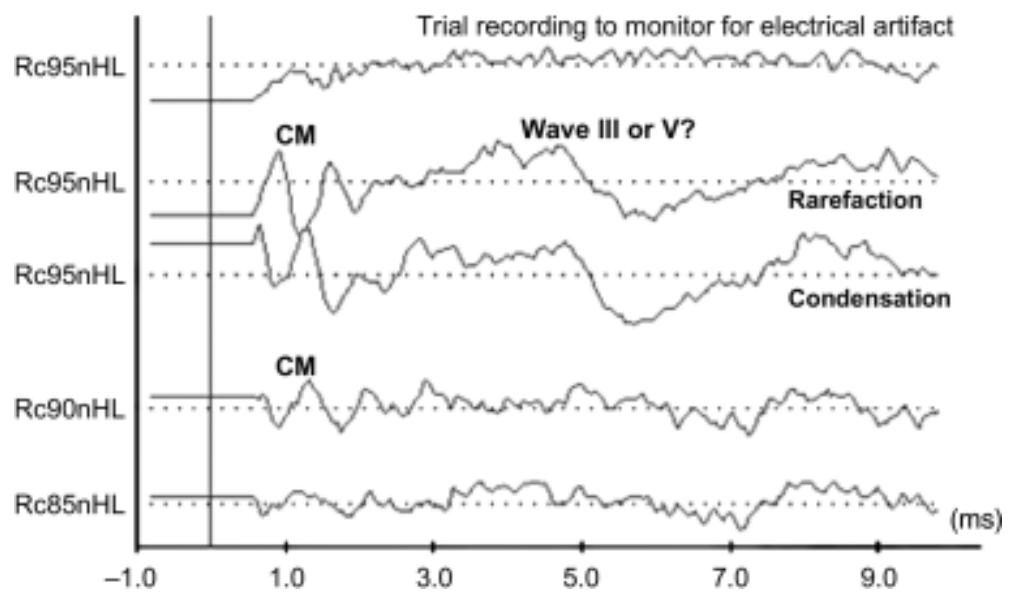

Fig. 2. Left ear ABR recordings: the top recording is the trial run to ensure that responses were not because of stimulus artifacts. The recordings below these indicate the presence of the cochlear microphonic response, which inverts with changing polarity (rarefaction and condensation). A neural response is observed at 95 and $90 \mathrm{~dB}$ but cannot be identified as either wave III or V.

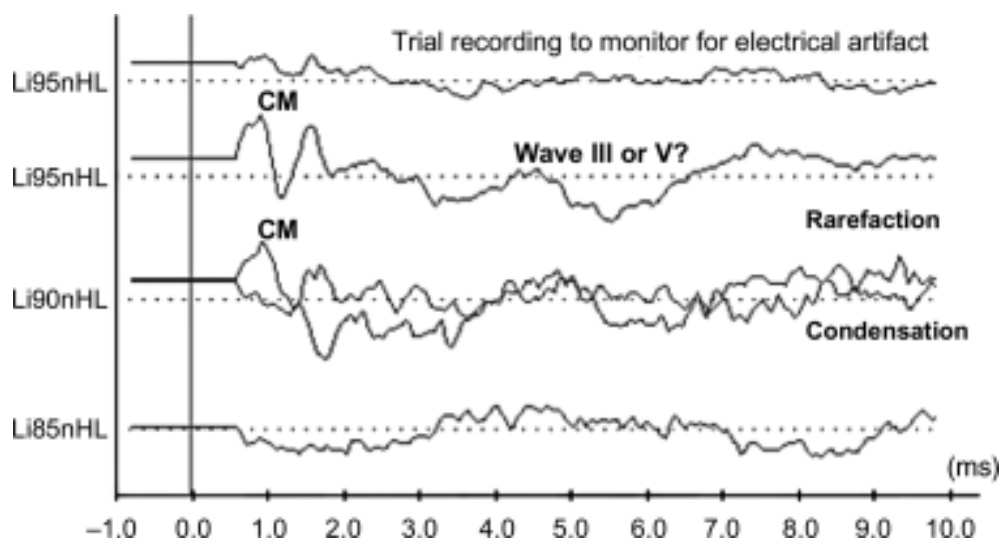




\section{References}

1. Niebuhr E. The cri du chat syndrome. Epidemiology, cytogenetics and clinical features. Hum Genet 1978: 44: 227-275.

2. Higurashi M, Oda M, Iijima K et al. Livebirths prevalence and follow-up of malformation syndromes in 27,472 newborns. Brain Dev 1990: 12: 770-773.

3. Wilkins LE, Brown JA, Nance WE et al. Clinical heterogeneity in 80 home-reared children with cri-du-chat syndrome. J Pediatr 1983: 102: 528-533.

4. Cerruti Mainardi P. Cri du chat syndrome. Orphanet J Rare Dis 2006: 1: 33.

5. Cerruti Mainardi P, Perfumo C, Calì A et al. Clinical and molecular characterization of 80 patients with 5p deletion: genotype-phenotype correlation. J Med Genet 2001: 38: $151-158$.

6. Baird SM, Campbell D, Ingram R et al. Young children with cri-du-chat: genetic, developmental, and behavioral profiles. Infant-Toddler Intervention: The Transdisc J 2001: 11 (1): 1-14.

7. Cornish KM, Pigram J. Developmental and behavioural characteristics of cri du chat syndrome. Arch Dis Child 1996: 75: 448-450.

8. Breg WR, Steele MW, Miller OJ et al. The cri du chat syndrome in adolescents and adults: clinical finding in 13 older patients with partial deletion of the short arm of chromosome $\mathrm{N}^{\circ} 5$ (5p-). J Pediatr 1970: 77: 782-791.

9. Campbell DJ, Carlin ME, Justen JE III et al. Cri-du-chat syndrome: a topical overview. 5p Minus Society 2004: 1: 1-7.

10. Baccichetti C, Lenzini E, Artifoni L et al. Terminal deletion of the short arm of chromosome 5. Clin Genet 1988: 34: 219-223.

11. Choong YF, Watts P, Little E et al. Goldenhar and cri-du-chat syndromes: a contiguous gene deletion syndrome? J AAPOS 2003: 7: 226-227.

12. Starr A, Picton TW, Sininger Y et al. Auditory neuropathy. Brain 1996: 119: 741753.

13. Rapin I, Gravel J. "Auditory neuropathy": physiologic and pathologic evidence calls for more diagnostic specificity. Int J Pediatr Otorhinolaryngol 2003: 67: 707-728.

14. Rance G, Beer DE, Cone-Wesson B et al. Clinical findings for a group of infants and young children with auditory neuropathy. Ear Hear 1999: 20: 238-252. 
15. Cornish KM, Munir F. Receptive and expressive speech skills in children with cridu-chat syndrome. J Commun Disord 1998: 31: 73-80.

16. Cornish KM, Bramble D, Munir F et al. Cognitive functioning in children with typical cri du chat (5p-) syndrome. Dev Med Child Neurol 1999: 41 (4): 263-266.

17. Stredler-Brown A. Developing a treatment program for children with auditory neuropathy. Semin Hear 2002: 23: 239-249.

18. Berlin CI, Li L, Hood LJ et al. Auditory neuropathy/dys-synchrony: after the diagnosis, then what? Semin Hear 2002: 23: 209-214.

19. Medina M, Marinescu RC, Overhauser J et al. Hemizigosity of delta-catenin (CTNND2) is associated with severe mental retardation in cri-du-chat syndrome. Genomics 2000: 63: 157-164.

20. Matsuda M, Keino H. Roles of $\beta$-catenin in inner ear development in rat embryos. Anat Emrbyol 2000 202: 39-48.

21. Takebayashi S, Nakagawa T, Kojima $\mathrm{K}$ et al. Expression of $\beta$-catenin in developing auditory epithelia of mice. Acta Otolaryngol 2004: 124 (S551): 18-21.

22. Takebayashi S, Nakagawa $\mathrm{T}$, Kojima $\mathrm{K}$ et al. Nuclear translocation of $\beta$-catenin in developing auditory epithelia of mice. Neuroreport 2005: 16 (5): 431-434. 